\title{
Microindentation Hardness of Protein Crystals under Controlled Relative Humidity
}

\author{
Takeharu Kishi ${ }^{1}$, Ryo Suzuki ${ }^{1}$, Chika Shigemoto ${ }^{1}$, Hidenobu Murata ${ }^{1}$, Kenichi Kojima ${ }^{2}$ \\ and Masaru Tachibana ${ }^{1, *}$ \\ 1 Graduate School of Nanobioscience, Yokohama City University, Yokohama 236-0027, Japan; \\ nano_solid@yahoo.co.jp (T.K.); n175302b@yokohama-cu.ac.jp (R.S.); \\ n175222f@yokohama-cu.ac.jp (C.S.); hmr@@yokohama-cu.ac.jp (H.M.) \\ 2 Department of Education, Yokohama Soei University, Yokohama 226-0015, Japan; kkojima@soei.ac.jp \\ * Correspondence: tachiban@yokohama-cu.ac.jp; Tel.: +81-45-787-2307
}

Academic Editors: Ronald W. Armstrong, Stephen M. Walley and Wayne L. Elban

Received: 9 October 2017; Accepted: 31 October 2017; Published: 4 November 2017

\begin{abstract}
Vickers microindentation hardness of protein crystals was investigated on the (110) habit plane of tetragonal hen egg-white lysozyme crystals containing intracrystalline water at controlled relative humidity. The time evolution of the hardness of the crystals exposed to air with different humidities exhibits three stages such as the incubation, transition, and saturation stages. The hardness in the incubation stage keeps a constant value of $16 \mathrm{MPa}$, which is independent of the humidity. The incubation hardness can correspond to the intrinsic one in the wet condition. The increase of the hardness in the transition and saturation stages is well fitted with the single exponential curve, and is correlated with the reduction of water content in the crystal by the evaporation. The saturated maximum hardness also strongly depends on the water content equilibrated with the humidity. The slip traces corresponding to the (11) [110] slip system around the indentation marks are observed in not only incubation but also saturation stages. It is suggested that the plastic deformation in protein crystals by the indentation can be attributed to dislocation multiplication and motion inducing the slip. The indentation hardness in protein crystals is discussed in light of dislocation mechanism with Peierls stress and intracrystalline water.
\end{abstract}

Keywords: protein crystal; lysozyme crystal; indentation; hardness; dislocation; intracrystalline water; relative humidity; Peierls stress; slip

\section{Introduction}

The knowledge of the mechanical properties of crystals is important for the elucidation of intra-crystalline bonds and practical issues such as the limits of mechanical stability [1,2]. The mechanical properties of protein crystals is greatly affected by water content, although dislocations still play a crucial role in plastic deformation. However, our understanding of the mechanical properties of protein crystals is poor compared with those for metal and covalent crystals. The reason is that most of the classical techniques developed for studying mechanical properties of metal solid appear inapplicable due to the small size and high fragility of protein crystals. On the other hand, there are interesting studies on the mechanical response to the hydration of biological materials such as bone by using micro- and nano-indentation techniques [3-6]. Such mechanical properties in hydrated biomaterials seem to be partially similar to those in protein crystals, although they are non-crystals.

Protein crystals are composed of huge protein molecules with irregular shapes. They also contain a large amount of water with 20 to 70 vol. $\%[7,8]$. These features are responsible for complex and weak intermolecular interactions in protein crystals. This also leads to the difficulty of protein crystallization [8]. On the other hand, it is expected that these features can lead to unique mechanical 
properties [9-11]. The intracrystalline water in protein crystals is qualitatively classified into two types: one is free water moving freely through the crystals and the other is bound water held around each protein molecule [12-14]. Especially, the free water can be easily evaporated when the crystals are exposed to open air. Thus the water content in the crystals is sensitive to the environmental condition such as relative humidity. The change in the water content affects the mechanical properties. Therefore, the experiments with controlled water content or relative humidity are required for not only accurate measurement but also understanding of water behavior in the crystals and the corresponding unique mechanical properties.

Most of the studies on the mechanical properties of protein crystals have been carried out for hen egg-white lysozyme (HEWL) crystals with polymorphisms such as tetragonal, orthorhombic, monoclinic, and triclinic forms. The pioneer studies on the elastic properties of cross-linked HEWL crystals had been carried out by Morozov and Morozova [15-17]. The dynamic elastic constants for native and gel-grown crystals containing sufficient intracrystalline water were measured in the ranges of $\mathrm{MHz}$ and $\mathrm{GHz}$ by the ultrasonic pulse-echo method [18-20] and the Brillouin scattering method [21-23], respectively. These measurements were carried out in the growth solution and the corresponding $98 \%$ relative humidity ( $\% \mathrm{RH})$. Almost all elastic constants for cross-linked tetragonal (T)- [24] and orthorhombic (O)-HEWL crystals [25] containing sufficient intracrystalline water at room temperature with $98 \% \mathrm{RH}$ were determined by the ultrasonic pulse-echo method. The value of $C_{11}$ of the normal elastic component in T-HEWL crystals is $5.50 \mathrm{GPa}$, which is almost equal to $5.24 \mathrm{GPa}$ of O-HEWL crystals. Note that these values are much lower than $12.99 \mathrm{GPa}$ of the bulk modulus of hydrated lysozyme molecule [20]. On the other hand, the $C_{44}$ of the shear component in O-HEWL crystals is $0.30 \mathrm{GPa}$, which is about a half as low as $0.68 \mathrm{GPa}$ of T-HEWL crystals. The change in the shear elastic constant seems to be correlated with the water contents of 39 and 43 vol. \% for native $\mathrm{T}$ - and O-HEWL crystals, respectively. Thus, the shear elastic constant in protein crystals is more sensitive to the water content than the normal one.

Furthermore, it was measured by the ultrasonic pulse-echo method that the normal and shear elastic constants of the T-HEWL crystals dried at $42 \% \mathrm{RH}$ are about 2 and 4 times as large as those in the wet condition with $98 \% \mathrm{RH}$, respectively [26]. A similar trend depending on the relative humidity has been also observed for dynamic elastic constants measured in the range of GHz by the Brillouin scattering method [22]. These results also mean that the magnitudes of the elastic constants, especially the shear component, in protein crystals strongly depend on water content associated with relative humidity. The shear elastic constant is strongly related to the characteristics of dislocations playing a crucial role in the plastic deformation. It is therefore suggested that the plastic deformation associated with dislocations is also more sensitive to water content than elastic properties.

The studies on plastic properties of protein crystals have been carried out by using Vickers microindentation method, mainly with T-HEWL ones [27-29]. In the wet condition, the indentation marks were clearly observed on the (110) crystal plane. Slip traces were also observed around the indentation. From the analysis of the slip traces, it has been shown that the plastic deformation is controlled by the dislocation mechanism with the $\{110\}\langle 110\rangle$ slip system. This has been also supported by the observation of slip dislocations by X-ray topography [30,31]. The average activation energy of the dislocation motion has been also evaluated to be $0.6 \mathrm{eV}$ from the measurements of the temperature dependence of the indentation hardness [28]. Furthermore, it has been found that the indentation hardness increases with the evaporation of the intracrystalline water in open air where the evaporation time dependence of the hardness has three stages such as incubation, transition, and saturation stages [29]. The maximum value of the hardness in the dried condition has been about one order of magnitude larger than that in the wet condition. Recently similar behaviors have been also observed for O-HEWL crystals [32]. However, these measurements have been carried out under ambient humidity. To clarify the hardness behavior, experiments under controlled relative humidity would be desirable. 
The plastic characteristics for glucose isomerase (GI), ferritin, trypsin, and insulin crystals besides HEWL ones have been also investigated by indentation method [33] and pushing method with a glass filament [34,35]. The unique plastic behavior such as creep was observed for GI crystals [33]. Additionally, extremely high quality GI crystals were clarified by X-ray topography with dislocation images and Pendellösung fringes [36]. On the other hand, a detailed mechanical response with anisotropic properties was simulated by using a continuum-based crystal plasticity model which was calibrated with Vickers microindentation hardness data [37]. This simulation with the hardness data enabled us to deduce the critical resolved shear stress on the slip plane of the T-HEWL crystals. Therefore, it is expected that the hardness measurements under controlled relative humidity can lead to more precise plastic characteristics.

In this paper we report the indentation hardness on the (110) habit plane of the T-HEWL crystals under controlled relative humidities. The time evolution of the hardness of the crystals exposed to air with different humidities exhibits three stages such as the incubation, transition, and saturation stages. The hardness in the incubation stage keeps a constant value of $16 \mathrm{MPa}$ which is independent of the humidity. The incubation hardness can correspond to the intrinsic one in the wet condition. The increase of the hardness in the transition and saturation stages is well fitted with a single exponential curve, and is correlated with the reduction of water content in the crystal by the evaporation. The saturated maximum hardness also strongly depends on the water content equilibrated with the humidity. The slip traces corresponding to the (110) [110] slip system around the indentation marks are observed in not only the incubation but also the saturation stages. It is suggested that the plastic deformation in the protein crystals by the indentation can be ascribed to dislocation multiplication and motion inducing the slip. The indentation hardness in the protein crystals is discussed in light of the dislocation mechanism with Peierls stress and intracrystalline water.

\section{Results and Discussion}

\subsection{Hardness at Controlled Humidity}

Figure 1 shows the time evolution of Vickers microindentation hardness on (110) habit plane of T-HEWL crystals at $296 \mathrm{~K}$ exposed to air with $35.9 \% \mathrm{RH}$. The hardness strongly depends on the exposure time to air. Note that the exposure of the crystal to air can lead to the evaporation of the intracrystalline water. The behavior of hardness exhibits three stages with increasing exposure time, as reported previously [29]. First stage is the incubation stage in which the magnitude of hardness keeps a constant value even during the water evaporation, where the indented plane is still kept in wet condition. Second stage is the transition stage in which the magnitude of hardness increases with increasing exposure time where the indented plane is partially dried. Third stage is the saturation one in which the magnitude of the hardness reaches a maximum value and almost keeps the value with increasing exposure time where the indented plane is highly dried. The maximum hardness can be controlled by the water content in the crystal equilibrated with the environmental condition such as temperature and humidity.

From data points in Figure 1, it is noted that the scatter of measured values in each stage, especially transition and saturation stages, is less than that reported elsewhere $[27,29]$. The low scattering of measured values is attributed to controlled relative humidity in this work. Thus, more accurate analysis of the hardness becomes possible. The value of the hardness in the incubation stage is found to be $16 \mathrm{MPa}$, as seen in Figure 1. This value is slightly lower than that reported previously [27-29]. The reason can be attributed to the high accuracy for the measurements at controlled humidity. The hardness of $16 \mathrm{MPa}$ is considered to be intrinsic incubation hardness in T-HEWL crystals containing sufficient intracrystalline water, although the origin for the incubation stage is discussed later.

Furthermore, it is found that the hardness curve in transition and saturation stages is well fitted with single exponential curve given by

$$
H_{v}=H_{v}^{\max }+A \exp \left(-k_{\mathrm{h}} t\right)
$$


where $H_{v}$ is Vickers microhardness, $k_{\mathrm{h}}$ is rate constant for the increase of the hardness, $t$ is exposure time, and $H_{v}^{\max }$ is saturated or maximum hardness. Note that a first data point with $H_{v}$ of more than $20 \mathrm{MPa}$ in the time evolution of the $H_{v}$, as shown in Figure 1, was defined as a starting point in the transition stage. From the fitting, we can evaluate $k_{\mathrm{h}}=0.027 \mathrm{~min}^{-1}$ and $H_{v}^{\max }=247.6 \mathrm{MPa}$.

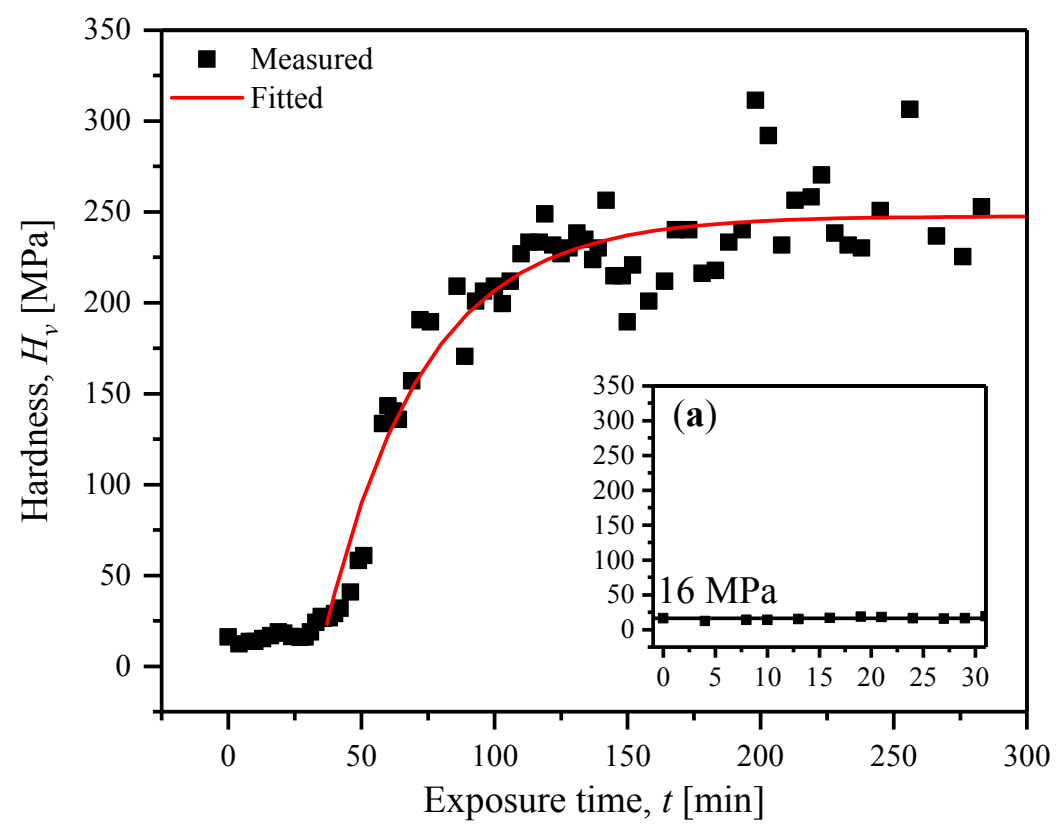

Figure 1. Time evolution of Vickers microindentation hardness on (110) habit plane of T-HEWL crystals at $296 \mathrm{~K}$ exposed to air with $35.9 \%$ relative humidity (RH). The hardness curve has three stages such as incubation, transition, and saturation with exposure time. The extended figure of the initial or incubation stage is shown in the inset. The fitting with single exponential curve is also drawn for the hardness curve in the transition and saturation stages.

Figure 2 shows the time evolution of Vickers microindentation hardness on (110) habit plane of T-HEWL crystals at $296 \mathrm{~K}$ exposed to air with different relative humidities such as $35.9,42.1,54.7$, 73.6, and $84.0 \% \mathrm{RH}$, where measured crystals have different sizes of 1.6, 7.5, 1.9, 1.8, and $1.7 \mathrm{~mm}^{3}$, respectively. All of hardness curves exhibit three stages such as incubation, transition, and saturation. At higher humidity of $84.0 \% \mathrm{RH}$, longer exposure time is required for the appearance of the saturation stage, as shown in Figure 2b. It should be noted that the magnitude of the hardness in the incubation stage is independent of relative humidity, and keeps a constant value, as shown in Figure 2c. This result is in good agreement with that in O-HEWL crystals reported recently [32]. Therefore, it is suggested that the hardness in the incubation stage corresponds to the intrinsic one of T-HEWL crystals with sufficient intracrystalline water in the wet condition as O-HEWL crystals.

The constant value of the hardness on (110) plane of T-HEWL crystals in the incubation stage is $16 \mathrm{MPa}$ even under different humidities, as seen in Figure 2c. The value is about two times as high as the average hardness of 7.8 MPa of O-HEWL crystals reported recently [32]. Actually, the hardness value of T-HEWL crystals in the incubation stage is higher than all values of 5.7, 8.1, and 9.6 MPa on (110), (010), and (011) crystal planes of O-HEWL crystals. The high hardness can be ascribed not only to the crystal form but also to the water content with 39 vol. \% in T-HEWL crystals smaller than 42 vol. \% in O-HEWL crystals as mentioned above.

For transition and saturation stages, all the hardness curves are well fitted with single exponential curves, as shown in Figure 2a,b. The $k_{\mathrm{h}}$ and $H_{v}^{\max }$ obtained by the fitting are presented in Table 1 . The rate constant, $k_{\mathrm{h}}$, depends on the relative humidity, as shown in Table 1 . The value of $k_{\mathrm{h}}$ increases with decreasing relative humidity. The value at $35.9 \%$ RH is $0.027 \mathrm{~min}^{-1}$, which is about seven times 
as high as $0.004 \mathrm{~min}^{-1}$ at $84.0 \% \mathrm{RH}$. The high $k_{\mathrm{h}}$ for the increase of the hardness can be attributed to the high evaporation rate of the intracrystalline water under low relative humidity.

(a)
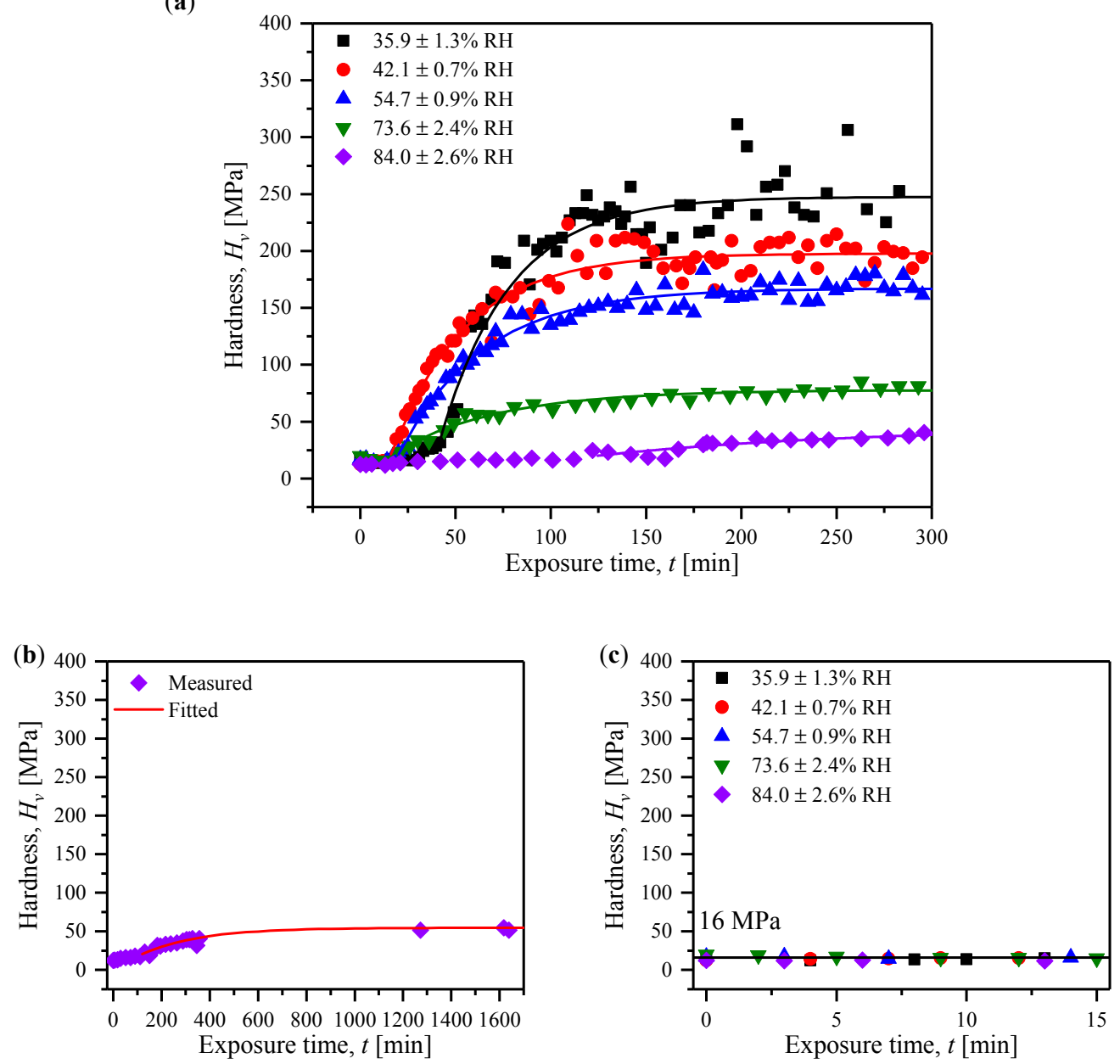

Figure 2. (a) Time evolution of Vickers microindentation hardness on (110) habit plane of T-HEWL crystals at $296 \mathrm{~K}$ exposed to air with different relative humidities such as 35.9, 42.1, 54.7, 73.6, and $84.0 \% \mathrm{RH}$. The hardness curve with longer exposure time at higher humidity of $84.0 \% \mathrm{RH}$ is shown (b). All hardness curves in $(\mathbf{a}, \mathbf{b})$ have three stages such as incubation, transition, and saturation ones with exposure time. The extended figure of the incubation stages at different relative humidities in (a) is shown in (c). The fittings with single exponential curves for the hardness curves in the transition and saturation stages are also drawn in $(\mathbf{a}, \mathbf{b})$.

Table 1. Rate constant, $k_{\mathrm{h}}$, for the increase of the hardness and maximum hardness, $H_{v}^{\max }$, in T-HEWL crystals under different relative humidities.

\begin{tabular}{ccc}
\hline Relative Humidity $[\% \mathbf{R H}]$ & $\boldsymbol{k}_{\mathbf{h}}\left[\mathbf{m i n}^{-\mathbf{1}}\right]$ & $\boldsymbol{H}_{\boldsymbol{v}}^{\max }[\mathbf{M P a}]$ \\
\hline 35.9 & 0.027 & 247.6 \\
42.1 & 0.026 & 197.8 \\
54.7 & 0.022 & 167.2 \\
73.6 & 0.018 & 77.8 \\
84.0 & 0.004 & 54.7 \\
\hline
\end{tabular}

Furthermore, it should be noted that the maximum hardness, $H_{v}^{\max }$, also strongly depends on the relative humidity, as presented in Table 1 . The value of $H_{v}^{\max }$ also increases with decreasing relative humidity. The value of $H_{v}^{\max }$ at $35.9 \% \mathrm{RH}$ is $247.6 \mathrm{MPa}$, which is about 5 times as high as $54.7 \mathrm{MPa}$ 
at $84.0 \% \mathrm{RH}$. The high $H_{v}^{\max }$ can be ascribed to the low water content by the evaporation of a large amount of intracrystalline water under low relative humidity. Thus, the $H_{v}^{\max }$ is controlled by the water content in the crystal equilibrated with environmental conditions such as relative humidity. Additionally, the hardness at $100 \% \mathrm{RH}$ is extrapolated from a fitted curve for the humidity dependence of the maximum hardness, as shown in Figure 3. The extrapolated value is $7 \mathrm{MPa}$, which is even lower than $16 \mathrm{MPa}$ in the incubation stage related to the wet condition, as mentioned above. The low value of the extrapolated hardness can correspond to real one of T-HEWL crystals in the solution at $100 \% \mathrm{RH}$.

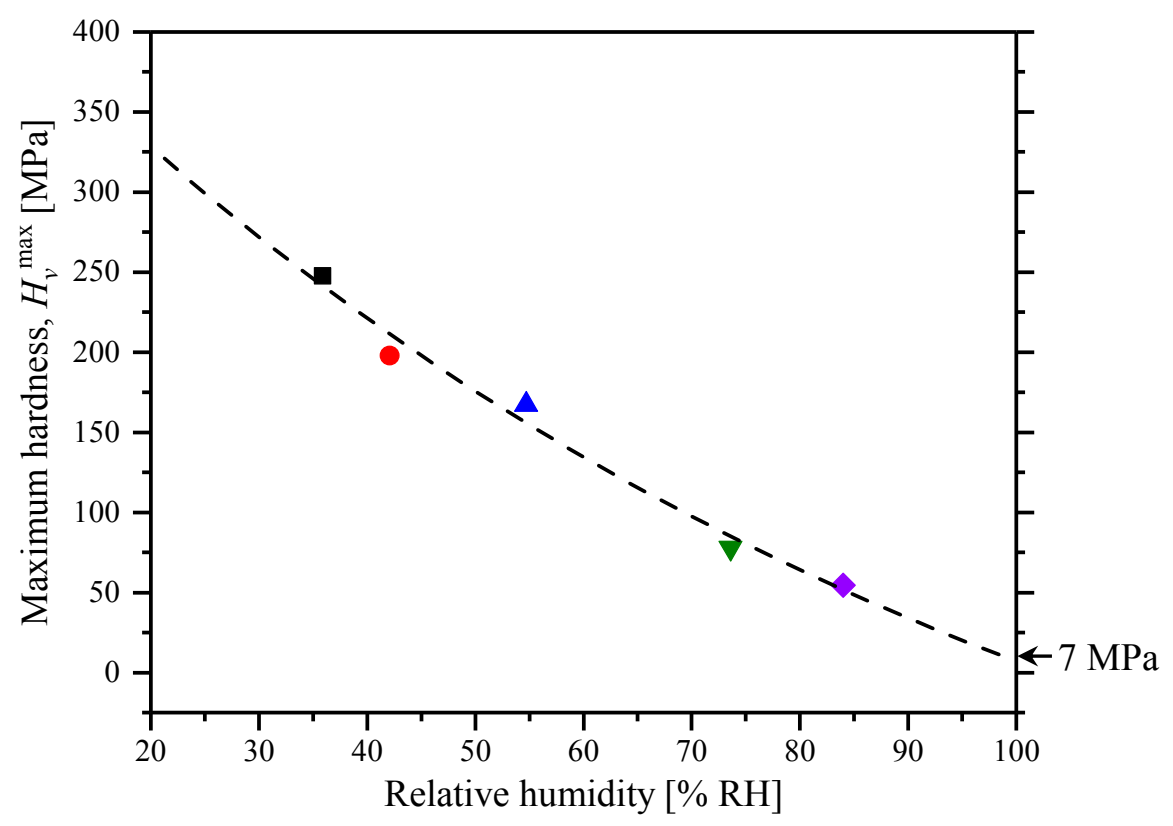

Figure 3. Humidity dependence of the maximum hardness, $H_{v}^{\max }$, obtained by the fitting with single exponential curve for the hardness curves in Figure 2a.

\subsection{Evaporation of Intracrystalline Water}

In order to know the behavior of water evaporation, the change in crystal weight of T-HEWL crystals exposed to different relative humidities was measured by using a thermogravimetric analyzer in which the humidity is controlled by the flow ratio of wet and dry $\mathrm{N}_{2}$ gases. Figure 4 a shows time evolutions of crystal weights of T-HEWL crystals at $296 \mathrm{~K}$ exposed to $\mathrm{N}_{2}$ gas with different relative humidities such as $39.1,55.7,74.3$, and $92.9 \% \mathrm{RH}$. Note that the crystal weight at $t=0$ corresponds to the sum of weights of intrinsic crystal with sufficient intracrystalline water and excess water around the crystal. As seen in Figure $4 a$, the crystal weight monotonically decreases with time evolution. The decay curves at 55.7, 74.3, and 92.9\% RH are well fitted with single exponential curves given by

$$
W=W_{0}+A \exp \left(-k_{\mathrm{w} 1} t\right)
$$

where $W$ is the relative weight to the initial one at $t=0, k_{\mathrm{w} 1}$ is first rate constant for the reduction of weight, i.e., the evaporation of water, and $t$ is the exposure time to $\mathrm{N}_{2}$ gas with controlled humidity. The typical fitting with single exponential curve for the measured decay curve at $92.9 \% \mathrm{RH}$ is shown in Figure $4 \mathrm{~b}$. The well-fitting by single exponential curve means that there is no significant change in the evaporation rate for the intracrystalline water and common water around the crystal. Namely, the characteristic of the evaporation of crystalline water, probably free water, is similar to that of common water. Additionally, the single exponential fitting is in good agreement with that in the hardness curves in transition and saturation stages, as shown in Figure 2. Thus, it is suggested that the change in the hardness in transition and saturation stages can be strongly correlated with the behavior of the evaporation of intracrystalline water, probably free water. The rate constant, $k_{\mathrm{w} 1}$, 
for the reduction of crystal weight, i.e., the evaporation of intracrystalline water, is estimated by the fitting for the decay curve. The values of $k_{\mathrm{w} 1}$ for the decay curves at different humidities in Figure 4 are presented in Table 2.
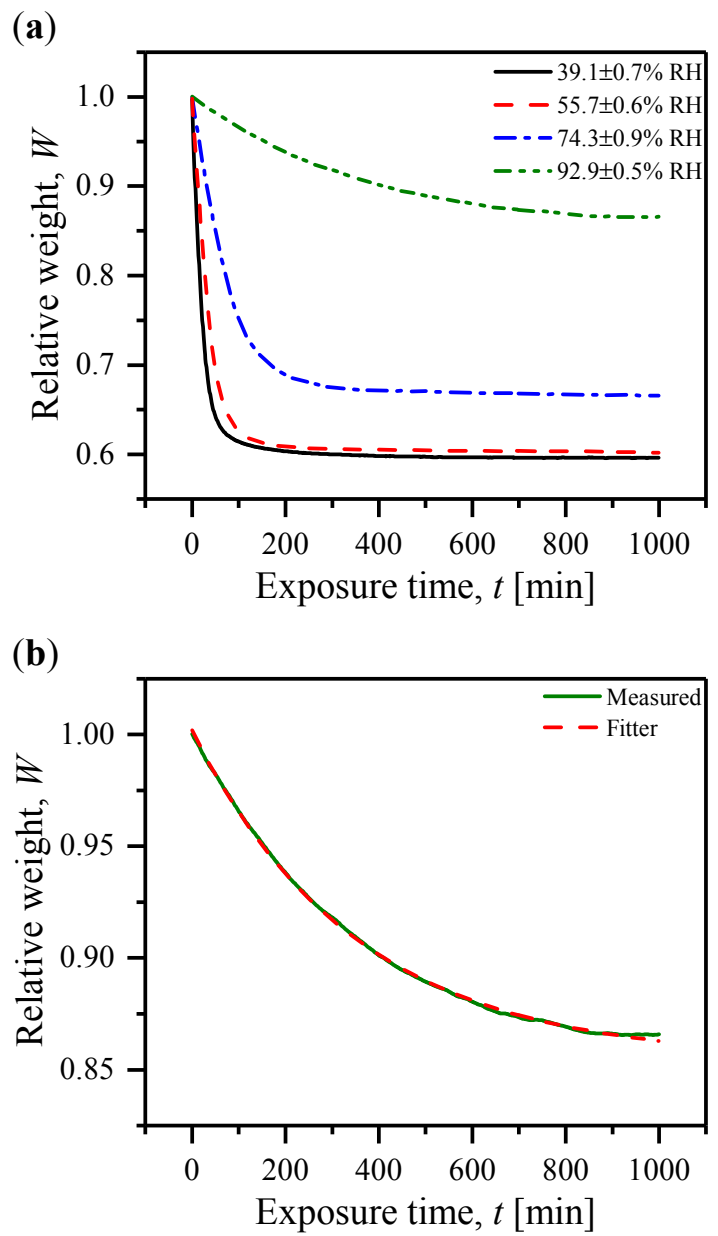

Figure 4. (a) Time evolution of the crystal weight of T-HEWL crystals at $296 \mathrm{~K}$ exposed to $\mathrm{N}_{2}$ gas with different relative humidities such as $39.1,55.7,74.3$, and $92.9 \% \mathrm{RH}$. The typical fitting with single exponential curve for the decay curve at $92.9 \% \mathrm{RH}$ is shown in (b).

Table 2. Rate constants of $k_{\mathrm{w} 1}$ and $k_{\mathrm{w} 2}$ for the reduction of crystal weights at different relative humidities.

\begin{tabular}{ccc}
\hline Relative Humidity [\%] & $\boldsymbol{k}_{\mathrm{w} \mathbf{1}}\left[\mathbf{m i n}^{-\mathbf{1}}\right]$ & $\boldsymbol{k}_{\mathrm{w} \mathbf{2}}\left[\mathrm{min}^{-\mathbf{1}}\right]$ \\
\hline 39.1 & 0.049 & 0.006 \\
55.7 & 0.029 & - \\
74.3 & 0.014 & - \\
92.9 & 0.003 & - \\
\hline
\end{tabular}

On the other hand, the decay curve at the lowest humidity of $39.1 \% \mathrm{RH}$ is well fitted not with single but with two exponential curves given by

$$
W=W_{0}+A_{1} \exp \left(-k_{\mathrm{w} 1} t\right)+A_{2} \exp \left(-k_{\mathrm{w} 2} t\right),
$$

where $k_{\mathrm{w} 2}$ is second rate constant for the reduction of crystal weight and $A_{1}$ and $A_{2}$ are the ratios of two kinds of evaporation processes with first and second rate constants, respectively. The clear difference in 
the fitting accuracy with single and two exponential curves is confirmed in Figure 5a,b. The well-fitting with two exponential curves means that the decay curve contains two kinds of evaporation processes with fast and slow rate constants. The two rate constants are also presented in Table 2. From comparing the values of rate constants in Table 2, fast evaporation process corresponding to $k_{\mathrm{w} 1}$ is observed in all humidities. As mentioned previously, there are two kinds of intracrystalline waters such as free water and bound water in protein crystals. It is therefore considered that the fast component, $k_{\mathrm{w} 1}$, is related to the evaporation of free water which can be easily evaporated through the crystal.

(a)

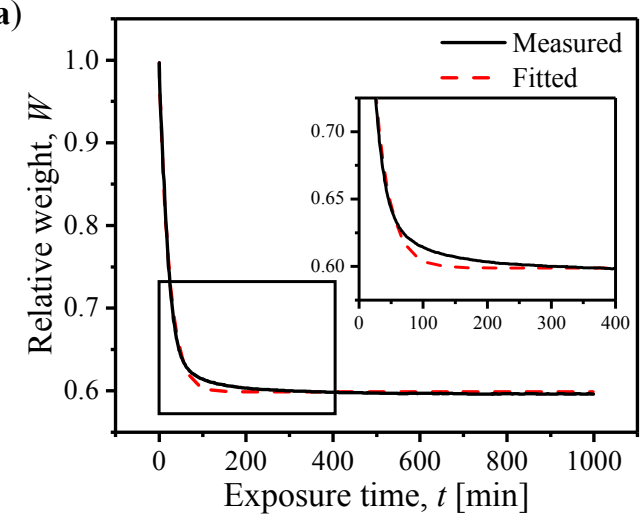

(b)

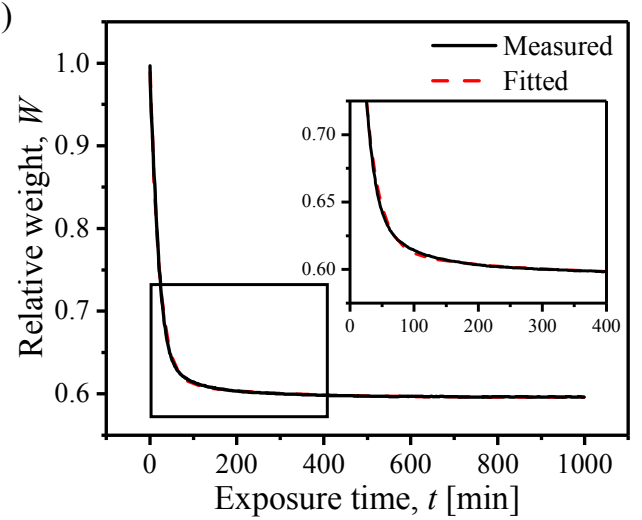

Figure 5. The decay curve of the crystal weight of T-HEWL crystals at 39.1\% RH in Figure 4 (a) and the corresponding fitting curves with single (a) and two exponential curves (b).

Figure 6 shows the fast rate constant, $k_{\mathrm{w} 1}$, for the reduction of crystal weight, i.e., the evaporation of free water, as a function of the relative humidity. The $k_{\mathrm{w} 1}$ increases with a decrease in the relative humidity. This means that the low humidity leads to the increase of the reduction rate of crystal weight, i.e., the evaporation rate of free water. The value of $k_{\mathrm{w} 1}$ at lowest humidity of $39.1 \% \mathrm{RH}$ is $0.049 \mathrm{~min}^{-1}$, which is larger by more than one order compared with $0.003 \mathrm{~min}^{-1}$ at highest humidity of $92.9 \% \mathrm{RH}$. This trend depending on the humidity is similar to the behavior of $k_{\mathrm{h}}$ for the increase of the hardness with decreasing humidity, as shown in Figure 6. On the other hand, the values of $k_{\mathrm{w} 1}$ for the reduction of crystal weight, especially for low humidities, are higher than those of $k_{\mathrm{h}}$ for the increase of the hardness, as seen in Figure 6. The discrepancy in the values might be due to the difference in humidity-control systems with dry-wet and $\mathrm{N}_{2}$ gas flow used in the hardness and crystal weight measurements, respectively.

The second, i.e., slow, rate constant, $k_{\mathrm{w} 2}$, of evaporation processes is observed at only lowest humidity of $39.1 \% \mathrm{RH}$. The slow component, $k_{\mathrm{w} 2}$, can be related to the evaporation of bound water around each protein molecule. Strictly bound water around protein molecules forms hydration layers $[12,14,38]$. The water in the outer layer is loosely bound to the protein compared with the inner layer. The loosely bound water can be evaporated at low humidity, although it is strongly bound with the protein compared with the free water. On the other hand, as shown in Figure 2, the hardness curve is well-fitted with single exponential one even under the lowest humidity. These results imply that the evaporation of the loosely bound water gives no significant effect on the behavior of the crystal hardness in this work.

As mentioned so far, the monotonical reduction of crystal weight, i.e., the evaporation of intracrystalline water, can explain the change in the hardness in the transition and saturation stages, whereas it cannot be simply correlated with a constant value of the hardness in the incubation stage. Now, let us consider the mechanism for the incubation. The intracrystalline water, mainly free water, is monotonically evaporated. On the other hand, the hardness first keeps a constant value in the incubation stage, as mentioned above. This means that the surface region corresponding to the indentation depth in the incubation stage is kept at the wet condition, although the intracrystalline 
water is monotonically evaporated. According to the studies on the drying mechanism in porous materials [39-41], the evaporation of water at the surface is followed by the flow of interior water to the surface. Similar process can occur in protein crystals with free water. When the evaporation rate of the surface water is equal to the diffusion rate of interior water to the surface, the crystal surface is always kept in the wet condition. Namely, the water content at the surface is kept at nearly constant, although the intracrystalline water is monotonically evaporated. Such equilibrium of evaporation and diffusion rates can be kept in high water content so that a constant value of the hardness at the wet condition appears as the incubation stage.

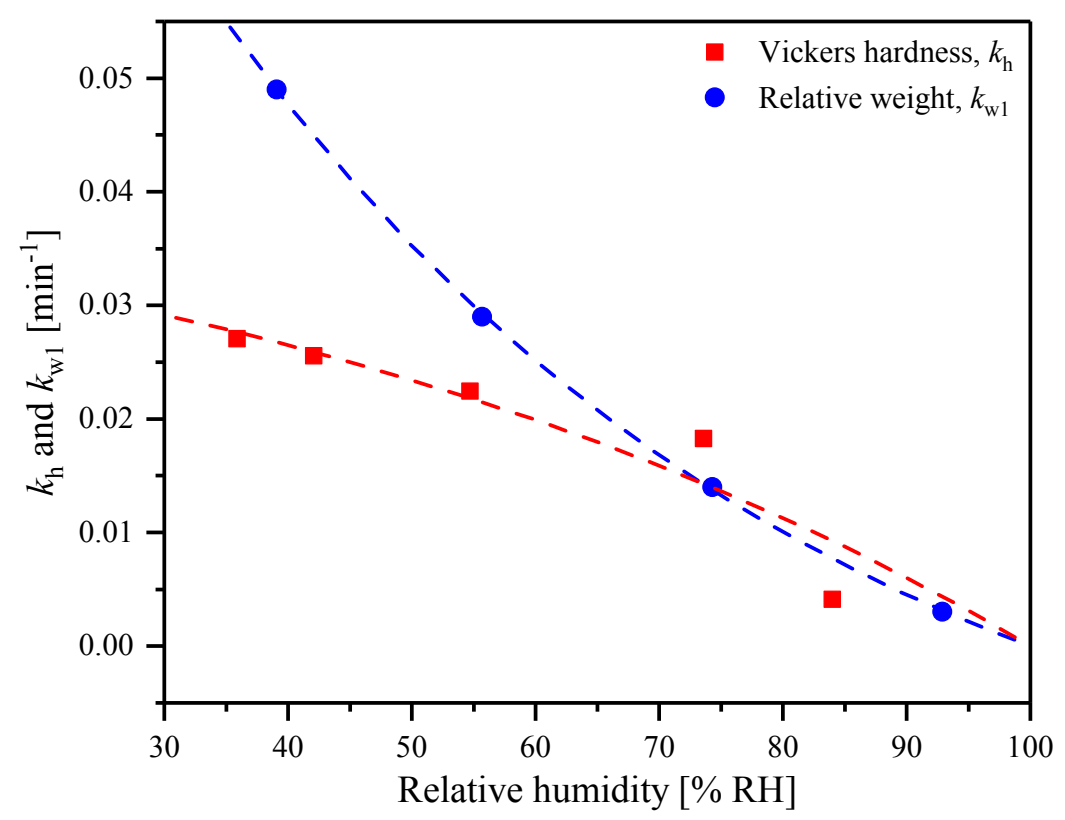

Figure 6. Comparison of rate constants of $k_{\mathrm{h}}$ and $k_{\mathrm{w} 1}$ for the increase of Vickers hardness and the reduction of crystal weight, respectively, as a function of the relative humidity.

Further reduction of water content leads to the decrease of the water evaporation and diffusion rates. Especially, the diffusion rate of interior water to the surface is more reduced compared with the evaporation demand after a critical water content in the crystal [42]. This reduction of diffusion rate leads to the drying at the indentation surface. As a result, the transition stage with the increase of the hardness appears with the drying. Thus, the constant hardness in the incubation stage can be explained based on the drying process in porous materials [39-41]. This also means that the drying mechanism of protein crystals with free water is similar to that of porous materials. Additionally, according to the drying process in porous materials $[39,40]$, the rapid drying before the incubation stage occurs, although it is actually difficult to measure it. Thus, real hardness of protein crystals in the solution becomes smaller than $16 \mathrm{MPa}$ in the incubation stage in Figure 2. This is consistent with the small hardness of $7 \mathrm{MPa}$ at $100 \% \mathrm{RH}$ extrapolated from the fitted curve for the humidity dependence of the $H_{v}^{\max }$ in Figure 3.

Such drying behavior in protein crystals affects the intermolecular interaction, e.g., lattice constant and elastic constant. The change in the intermolecular interaction greatly influences the dislocation mechanism, playing a crucial role in the plastic deformation.

\subsection{Dislocations and Peierls Stress}

Figure 7 shows indentation marks formed on (110) planes in the three stages. As seen in Figure 7a, the slip traces indicated by arrows around the indentation mark are clearly observed in the incubation stage related to the wet condition, as reported previously [27-29]. It is suggested that 
plastic deformation brought about by indentation mainly results from dislocation multiplication and motion, inducing the slip in the crystal. On the other hand, no clear slip trace around the indentation mark has been observed in the saturation stage related to the dried condition so far. This might be attributed to small plastic deformation corresponding to small indentation mark due to the high hardness in the saturation stage. In this work, the indentions with higher loads were also applied in the saturation stage. As a result, the clear slip traces around the larger indentation marks were sometimes observed even in the saturation stage related to dried condition as seen in Figure $7 \mathrm{~d}$. These results suggest that dislocation multiplication and motion can occur for the plastic deformation in all stages. However, actually, it is still difficult to observe the slip traces around the indentation marks even by high load indentation. This might be related to the poor crystal quality in the saturation stage, i.e., dried condition.

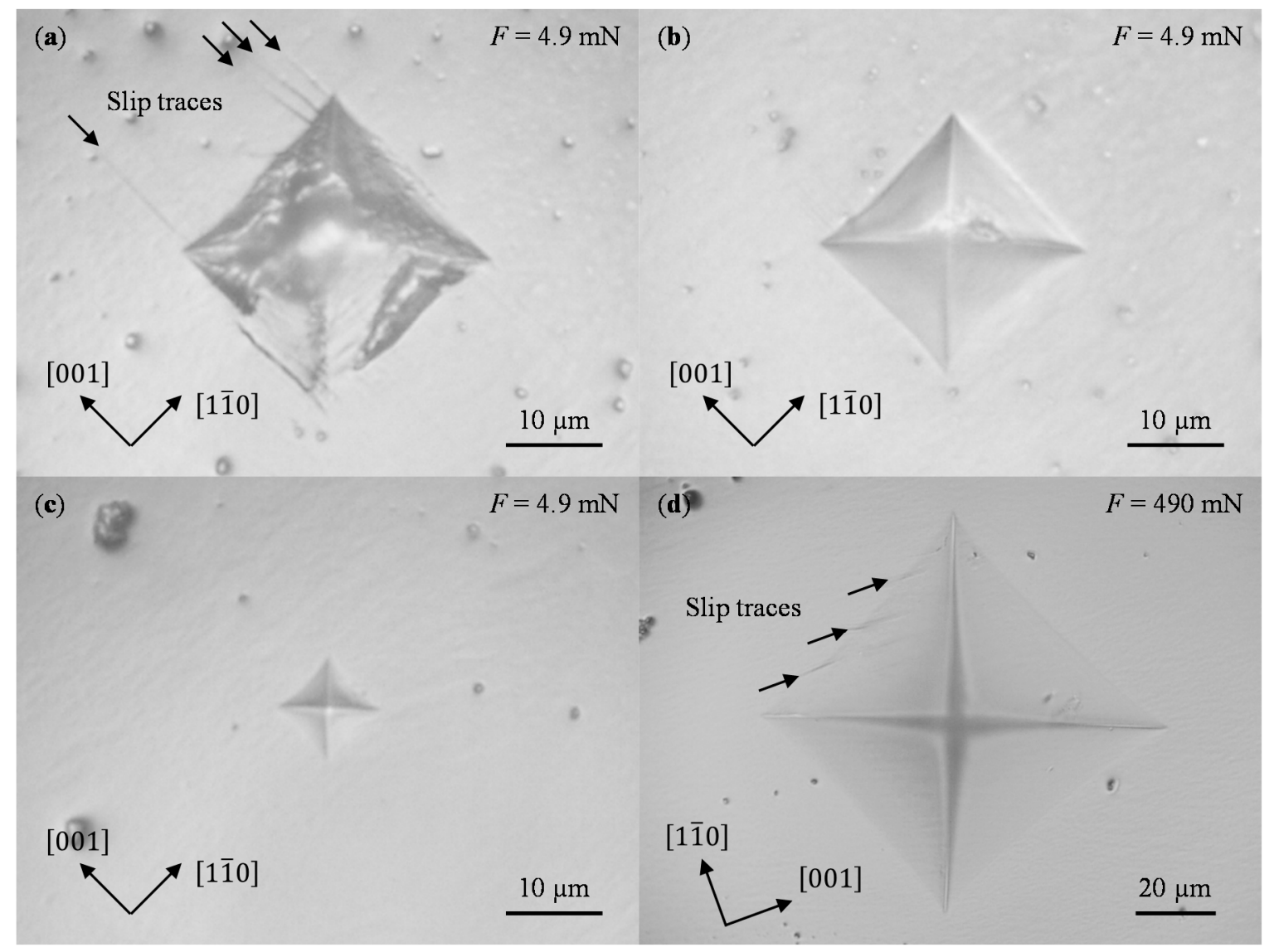

Figure 7. The morphologies around the indentation marks formed by the indentations on (110) habit planes of T-HEWL crystals in (a) incubation, (b) transition, and (c,d) saturation stages at $64 \% \mathrm{RH}$. Note that a load of $4.9 \mathrm{mN}(0.5 \mathrm{~g}$ weight) was used in $(\mathbf{a}-\mathbf{c})$, whereas a higher load of $490 \mathrm{mN}$ (50 g weight) was employed in (d). The slip traces are indicated by arrows in (a,d).

The surface morphology inside the indentation mark in the incubation stage is rough compared with the smooth surface in the transition and saturation stages, as seen in Figure 7. Additionally, the edges of the indentation mark in the incubation stage are partially disturbed in contrast to the sharp edges in another stages. Such roughness of the indentation mark can be ascribed to the pull-out effect due to the adhesion depending on the hydration by the indenter [43-45]. The correction of the adhesion on protein crystals by the indenter would be required for more accurate analysis of the indentation hardness.

The directions of all slip traces indicated by arrows in Figure 7a,d are parallel to $\langle 001\rangle$, as reported previously [27-29]. According to the dislocation self-energy in previous papers [28], $\{110\}\langle 001\rangle$ 
$(b=3.79 \mathrm{~nm})$ and $\{110\}\langle 110\rangle(b=11.1 \mathrm{~nm})$ are suggested as possible slip systems corresponding to $\langle 001\rangle$ slip traces, where $b$ is the magnitude of Burgers vector. However, the main slip system of $(1 \overline{1} 0)\langle 001\rangle$ does not appear on the (110) surface, since the (110) plane contains the $\langle 001\rangle$ axis. On the other hand, when the secondary slip system of (110) [110] is active, the slip traces of $\langle 001\rangle$ directions can be observed on the (110) plane. Thus, the slip traces observed near the indentation marks on the (110) surface, as seen in Figure 7a,d, correspond to secondary slip systems of (1110) [110].

Finally let us consider the mechanism of plastic deformation and hardness in protein crystals by indentation. The applied stress due to the indenter is concentrated in the indentation region and rapidly decreases away from it. When the indenter contacts the specimen surface, dislocations are generated beneath the indenter where the stress is very high. Then, the generated dislocations are able to move away from the indented region and thus T-HEWL crystals can deform plastically. Generally speaking, it is difficult to describe quantitatively the hardness value of crystals in terms of dislocation mechanism because the stress distribution around an indentation is very complicated. Peierls stress required to make a dislocation move in the crystal is estimated, although it cannot be directly related to the hardness. To evaluate the Peierls stress, we use a simple form of classic Peierls stress $[46,47]$ given by

$$
\sigma=\frac{2 G}{1-v} \exp \left(\frac{-2 \pi d}{b(1-v)}\right)
$$

where $G$ is the shear modulus, $v$ is Poisson's ratio, $d$ is the distance between slip planes, and $b$ is the magnitude of Burgers vector. In this work, the Peierls stresses are evaluated for $(1 \overline{1} 0)[110]$ $(b=11.1 \mathrm{~nm})$ slip system experimentally observed in both wet and dried T-HEWL crystals in the incubation and saturation stages, respectively, as seen in Figure 7. The Peierls stress of wet or hydrated T-HEWL crystals at $98 \% \mathrm{RH}$ is estimated to be $10.7 \mathrm{MPa}$ with $G=0.70 \mathrm{GPa}, v=0.42$, $d=5.59 \mathrm{~nm}$, and $b=11.1 \mathrm{~nm}$ where those values used in the calculation are experimental ones obtained from the measurements of sound velocities and X-ray diffractions of hydrated T-HEWL crystals at 98\% RH, reported previously [24]. On the other hand, the Peierls stress of dried or dehydrated T-HEWL crystals at $42 \% \mathrm{RH}$ is evaluated to be $57.2 \mathrm{MPa}$ with $G=2.64 \mathrm{GPa}, v=0.37, d=5.23 \mathrm{~nm}$, and $b=10.5 \mathrm{~nm}$. Note that those values used in the calculation are also experimental ones obtained from the measurements of sound velocities and X-ray diffractions of dehydrated T-HEWL crystals at $42 \% \mathrm{RH}$, reported previously [26]. The value of Peierls stress at $42 \% \mathrm{RH}$ is $57.2 \mathrm{MPa}$, which is about six times as high as $10.7 \mathrm{MPa}$ at $98 \% \mathrm{RH}$. This trend depending on the humidity is in good agreement with the increase of one order of the hardness experimentally observed at $42 \% \mathrm{RH}$, as shown in Figure 2 . Additionally, the values of the Peierls stress are similar order to 16 and $198 \mathrm{MPa}$ in the incubation and saturation stages at $42 \% \mathrm{RH}$, respectively, as shown in Figure 2. Thus, it is suggested that the hardness in protein crystals can be comparably correlated with Peierls stress based on simple model as typical metal and covalent crystals.

\section{Materials and Methods}

Three times crystallized HEWL (Wako Pure Chemical Industries, Ltd., Osaka, Japan) was used without further purification. T-HEWL crystals $\left(P 4_{3} 2_{1} 2, a=b=7.91 \mathrm{~nm}, c=3.79 \mathrm{~nm}, Z=8\right)$ were grown by means of a salt-concentration gradient method at $296 \mathrm{~K}$ in test tubes held vertically and using $\mathrm{NiCl}_{2}$ as a precipitant [48]. Large crystals up to a size of $5 \mathrm{~mm}$ were grown over two weeks. Almost all the crystals had habit plane such as $\{110\}$ and $\{101\}$. In this experiment, T-HEWL crystals with (110) habit plane of approximately $2 \times 2 \mathrm{~mm}^{2}$ were used for the measurements of Vickers hardness and crystal weight.

The Vickers hardness, $H_{v}$, was measured by using a microindentation testing machine (HM-221, Mitutoyo Co., Kawasaki, Japan). In order to measure the hardness at controlled relative humidity, the testing machine was covered with a simplified plastic chamber with $12.1 \times 10^{-3} \mathrm{~m}^{3}$. The relative humidity in the chamber is controlled by using water, silica-gel (Wako Pure Chemical Industries, Ltd., Osaka, Japan), and humidity control agents (DRY WET, Toshin Chemicals Co., Tokyo, Japan). 
The lower humidity of $35.9 \%$ RH was controlled by using the silica-gel (190 g). The middle humidity of 42.1 and $54.7 \%$ RH is realized by using the DRY WET $(40 \mathrm{~g})$. The higher humidities of 73.6 and $84.0 \% \mathrm{RH}$ were reached by using water $(300 \mathrm{~mL})$. Note that the controlled humidity in the chamber slightly depended on the outside humidity, since the simplified chamber had a little leak from the outside. The time evolution of the hardness was measured at $296 \mathrm{~K}$ in air with the controlled relative humidities. Just after the crystal is transferred from solution on the indentation stage in open air, the crystal plane is covered with solution droplet. In that situation, it is difficult to indent the crystal plane and/or observe the indentation marks. The clear indentation marks are confirmed after a few minutes with the evaporation of water. That time when the first indentation mark is observed is defined as $t=0$ of exposure time to air.

The indentation was carried out on (110) habit planes of T-HEWL crystals. The indenter, with a load of $4.9 \mathrm{mN}$ ( $0.5 \mathrm{~g}$ weight) and $490 \mathrm{mN}$ (50 g weight), was pulled down to the crystal plane at a velocity of $0.01 \mathrm{mms}^{-1}$. The contact period of the indenter with the plane was $5 \mathrm{~s}$, which is hold time at maximum load. The indentation marks were observed by using an optical microscope with a magnification of 100. In this experiment, the length of the diagonal of the indentation mark was approximately $20 \mu \mathrm{m}$ in the incubation stage. The distance between the indentation marks and crystal edges was $50 \mu \mathrm{m}$ at least. The separation of the indentation marks is more than the same length of indentation marks at least. It is difficult to separate the indentation marks with longer length since the area of the indentation marks is limited due to the small crystal. A standard block of hardness (HV700, Yamamoto Scientific Tool Laboratory, Funabashi, Japan) was used for calibration of the microindentation testing machine. The $H_{v}$ was determined with equation $1.854\left(F d^{-2}\right)$, where $F(\mathrm{~N})$ and $d(\mathrm{~mm})$ are the load and average length of the diagonal of the indentation mark, respectively. Note that the hardness, as evaluated above, would include the error of $10 \%$ at least assuming the error of $1 \mu \mathrm{m}$ in the measured value of the diagonal of $d=20 \mu \mathrm{m}$.

The weight measurement was carried out at $296 \mathrm{~K}$ by using a thermogravimetric analyzer (STA7000, Hitachi High-Technologies Co., Tokyo, Japan). In this analyzer, the relative humidity was controlled by a gas mixture of dry and wet $\mathrm{N}_{2}$ gases with controlled water vapor. The flow rates of dry and wet $\mathrm{N}_{2}$ gases were 200 and $100 \mathrm{~mL} / \mathrm{min}$, respectively.

\section{Conclusions}

We have shown the indentation hardness of T-HEWL crystals with intracrystalline water under controlled relative humidities. The hardness strongly depends on the water content in the crystals associated with the evaporation and humidity. The evaporation process is similar to that in porous materials. The slip traces related to dislocations multiplication and motion are clearly observed around the indentation marks. The hardness and plastic deformation in protein crystals by the indentation can be explained by the dislocation mechanism with Peierls stress and the change in the water content. The knowledge of such a dehydration process on the hardness of protein crystals is useful for the elucidation of not only the fundamental interest but also various applications and practical issues such as the handing of the protein crystals, e.g., substrate or drug binding, heavy-atom compound binding, and cryoprotectant soaks.

Acknowledgments: This work was supported in part by KAKENHI Grant-in-Aid for Scientific Research (C) (No. 25420694 and 16K06708).

Author Contributions: Takeharu Kishi, Hidenobu Murata, and Masaru Tachibana conceived and designed the experiments; Takeharu Kishi, Ryo Suzuki, and Chika Shigemoto performed the experiments; all authors analyzed the data; Takeharu Kishi, Ryo Suzuki, Chika Shigemoto, Kenichi Kojima, and Masaru Tachibana wrote the paper.

Conflicts of Interest: The authors declare no conflict of interest. 


\section{References}

1. Meyers, M.A.; Chawla, K.K. Mechanical Behavior of Materials, 2nd ed.; Cambridge University Press: Cambridge, UK, 2008; ISBN 9780521866750.

2. Gilman, J.J. Chemistry and Physics of Mechanical Hardness, 1st ed.; John Wiley and Sons: Hoboken, NJ, USA, 2009; ISBN 9780470226520.

3. Bembey, A.K.; Oyen, M.L.; Bushby, A.J.; Boyde, A. Viscoelastic properties of bone as a function of hydration state determined by nanoindentation. Philos. Mag. 2006, 86, 5691-5703. [CrossRef]

4. Bembey, A.K.; Bushby, A.J.; Boyde, A.; Ferguson, V.L.; Oyen, M.L. Hydration effects on the micro-mechanical properties of bone. J. Mater. Res. 2006, 21, 1962-1968. [CrossRef]

5. Oyen, M.L. Poroelastic nanoindentation responses of hydrated bone. J. Mater. Res. 2008, 23, 1307-1314. [CrossRef]

6. Oyen, M.L. Nanoindentation of hydrated materials and tissues. Curr. Opin. Solid. State Mater. Sci. 2015, 19, 317-323. [CrossRef]

7. McPherson, A. Crystallization of Biological Macromolecules; Cold Spring Harbor Laboratory Press: New York, NY, USA, 1999; ISBN 9780879695279.

8. Matthews, B.W. Solvent content of protein crystals. J. Mol. Biol. 1968, 33, 491-497. [CrossRef]

9. Vilenchik, L.Z.; Griffith, J.P.; Clair, N.S.; Navia, M.A.; Margolin, A.L. Protein crystals as novel microporous materials. J. Am. Chem. Soc. 1998, 120, 4290-4294. [CrossRef]

10. Margolin, A.L.; Navia, M.A. Protein crystals as novel catalytic materials. Angew. Chem. Int. Ed. 2001, 40, 2204-2222. [CrossRef]

11. Abe, S.; Ueno, T. Design of protein crystals in the development of solid biomaterials. RSC Adv. 2015, 5, 21366-21375. [CrossRef]

12. Otting, G.; Liepinish, E.; Wuthrich, K. Protein hydration in aqueous solution. Science 1991, 254, $974-980$. [CrossRef] [PubMed]

13. Morozov, V.N.; Kachalova, G.S.; Evtodienko, V.U.; Lanina, N.F.; Morozova, T.Y. Permeability of lysozyme tetragonal crystals to water. Eur. Biophys. J. 1995, 24, 93-98. [CrossRef]

14. Jones, M.J.; Ulrich, J. Are different protein crystal modifications polymorphs? A discussion. Chem. Eng. Technol. 2010, 33, 1571-1576. [CrossRef]

15. Morozov, V.N.; Morozova, T.Y. Viscoelastic properties of protein crystals: Triclinic crystals of hen egg white lysozyme in different conditions. Biopolymers 1981, 20, 451-467. [CrossRef] [PubMed]

16. Morozov, V.N.; Morozova, T.Y.; Kachalova, G.S.; Myachin, E.T. Interpretation of water desorption isotherms of lysozyme. Int. J. Biol. Macromol. 1988, 10, 329-336. [CrossRef]

17. Zenchenko, T.A.; Pozharskii, E.V.; Morozov, V.N. A magnetic micromethod to measure Young's modulus of protein crystals and other polymer materials. J. Biochem. Biophys. Methods 1996, 33, 207-215. [CrossRef]

18. Tachibana, M.; Kojima, K.; Ikuyama, R.; Kobayashi, Y.; Ataka, M. Sound velocity and dynamic elastic constants of lysozyme single crystals. Chem. Phys. Lett. 2000, 332, 259-264. [CrossRef]

19. Tachibana, M.; Kojima, K.; Ikuyama, R.; Kobayashi, Y.; Ataka, M. Erratum to: Sound velocity and dynamic elastic constants of lysozyme single crystals. Chem. Phys. Lett. 2002, 354, 360. [CrossRef]

20. Tachibana, M.; Koizumi, H.; Kojima, K. Effect of intracrystalline water on longitudinal sound velocity in tetragonal hen-egg-white lysozyme crystals. Phys. Rev. E 2004, 69, 051921. [CrossRef] [PubMed]

21. Caylor, C.L.; Speziale, S.; Kriminski, S.; Duffy, T. Measuring the elastic properties of protein crystals by Brillouin scattering. J. Cryst. Growth 2001, 232, 498-501. [CrossRef]

22. Speziale, S.; Jiang, F.; Caylor, C.L.; Kriminski, S.; Zha, C.S.; Thorne, R.E.; Duffy, T.S. Sound velocity and elasticity of tetragonal lysozyme crystals by Brillouin spectroscopy. Biophys. J. 2003, 85, 3202-3213. [CrossRef]

23. Hashimoto, E.; Aoki, Y.; Seshimo, Y.; Sasanuma, K.; Ike, Y.; Kojima, S. Dehydration process of protein crystals by micro-brillouin scattering. Jpn. J. Appl. Phys. 2008, 47, 3839-3842. [CrossRef]

24. Koizumi, H.; Tachibana, M.; Kojima, K. Elastic constants in tetragonal hen egg-white lysozyme crystals containing large amount of water. Phys. Rev. E 2009, 79, 061917. [CrossRef] [PubMed]

25. Kitajima, N.; Tsukashima, S.; Fujii, D.; Tachibana, M.; Koizumi, H.; Wako, K.; Kojima, K. Elastic constants in orthorhombic hen egg-white lysozyme crystals. Phys. Rev. E 2014, 89, 012714. [CrossRef] [PubMed] 
26. Koizumi, H.; Tachibana, M.; Kojima, K. Observation of all the components of elastic constants using tetragonal hen egg-white lysozyme crystals dehydrated at 42\% relative humidity. Phys. Rev. E $2006,73$. [CrossRef] [PubMed]

27. Tachibana, M.; Kobayashi, Y.; Shimazu, T.; Ataka, M.; Kojima, K. Growth and mechanical properties of lysozyme crystals. J. Cryst. Growth 1999, 198, 661-664. [CrossRef]

28. Koizumi, H.; Tachibana, M.; Kawamoto, H.; Kojima, K. Temperature dependence of microhardness of tetragonal hen-egg-white lysozyme single crystals. Philos. Mag. 2004, 84, 2961-2968. [CrossRef]

29. Koizumi, H.; Kawamoto, H.; Tachibana, M.; Kojima, K. Effect of intracrystalline water on micro-Vickers hardness in tetragonal hen egg-white lysozyme single crystals. J. Phys. D 2008, 41, 074019. [CrossRef]

30. Tachibana, M.; Koizumi, H.; Izumi, K.; Kajiwara, K.; Kojima, K. Identification of dislocations in large tetragonal hen egg-white lysozyme crystals by synchrotron white-beam topography. J. Synchrotron Radiat. 2003, 10, 416-420. [CrossRef] [PubMed]

31. Mukobayashi, Y.; Kitajima, N.; Yamamoto, Y.; Kajiwara, K.; Sugiyama, H.; Hirano, K.; Kojima, K.; Tachibana, M. Observation of dislocations in hen egg-white lysozyme crystals by synchrotron monochromatic-beam X-ray topography. Phys. Stat. Sol. 2009, 206, 1825-1828. [CrossRef]

32. Suzuki, R.; Kishi, T.; Tsukashima, S.; Tachibana, M.; Wako, K.; Kojima, K. Hardness and slip systems of orthorhombic hen egg-white lysozyme crystals. Philos. Mag. 2016, 96, 2930-2942. [CrossRef]

33. Tait, S.; White, E.T.; Litster, J.D. Mechanical characterization of protein crystals. Part. Part. Syst. Charact. 2008, 25, 266-276. [CrossRef]

34. Nanev, C.N.; Dimitrov, I.; Tsekova, D. Adhesion of protein crystals: Measurement of the detachment force. Cryst. Res. Technol. 2006, 41, 505-509. [CrossRef]

35. Nanev, C.N. Brittleness of protein crystals. Cryst. Res. Technol. 2012, 47, 922-927. [CrossRef]

36. Suzuki, R.; Koizumi, H.; Kojima, K.; Fukuyama, S.; Arai, Y.; Tsukamoto, K.; Suzuki, Y.; Tachibana, M. Characterization of grown-in dislocations in high-quality glucose isomerase crystals by synchrotron monochromatic-beam X-ray topography. J. Cryst. Growth 2017, 468, 299-304. [CrossRef]

37. Zamiri, A.; De, S. Modeling the mechanical response of tetragonal lysozyme crystals. Langmuir 2010, 26, 4251-4257. [CrossRef] [PubMed]

38. Pal, S.K.; Zewail, A.H. Dynamics of water in biological recognition. Chem. Rev. 2004, 104, $2099-2123$. [CrossRef] [PubMed]

39. Bray, Y.L.; Prat, M. Three-dimensional pore network simulation of drying in capillary porous media. Int. J. Heat Mass Tran. 1999, 42, 4207-4224. [CrossRef]

40. Yiotis, A.G.; Tsimpanogiannis, I.N.; Stubos, A.K.; Yortsos, Y.C. Pore-network study of the characteristic periods in the drying of porous materials. J. Colloid. Interface Sci. 2006, 297, 738-748. [CrossRef] [PubMed]

41. Lehmann, P.; Assouline, S.; Or, D. Characteristic lengths affecting evaporative drying of porous media. Phys. Rev. E 2008, 77. [CrossRef] [PubMed]

42. Yiotis, A.G.; Salin, D.; Tajer, E.S.; Yortsos, Y.C. Drying in porous media with gravity-stabilized fronts: Experimental results. Phys. Rev. E 2012, 86. [CrossRef] [PubMed]

43. Ebenstein, D.M.; Pruitt, L.A. Nanoindentation of biological materials. Nano Today 2006, 1, 26-33. [CrossRef]

44. Ferguson, V.L. Deformation partitioning provides insight into elastic, plastic, and viscous contributions to bone materials behavior. J. Mech. Behav. Biomed. 2009, 2, 364-374. [CrossRef] [PubMed]

45. Oyen, M.L. Nanoindentation of biological and biomimetic materials. Exp. Tech. 2013, 37, 73-87. [CrossRef]

46. Hirth, J.P.; Lothe, J. Theory of Dislocations, 2nd ed.; Wiley: Hoboken, NY, USA, 1982; ISBN 9780894646171.

47. Hull, D.; Bacon, D.J. Introduction to Dislocations, 5th ed.; Butterworth-Heinemann: Oxford, UK, 2011; ISBN 9780080966724.

48. Tachibana, M.; Kojima, K. Growth, Defects and mechanical properties of protein single crystals. Curr. Top. Cryst. Growth Res. 2002, 6, 35-49.

(C) 2017 by the authors. Licensee MDPI, Basel, Switzerland. This article is an open access article distributed under the terms and conditions of the Creative Commons Attribution (CC BY) license (http:/ / creativecommons.org/licenses/by/4.0/). 